\title{
Duality in the Flat Cohomology of Curves
}

\author{
M. Artin (Cambridge, Mass.)^ and J.S. Milne (Ann Arbor, Mich.)`
}

To Jean-Pierre Serre

\section{Contents}

1. The First Exact Sequence . . . . . . . . . . . . . . . . . . 113

2. The Second Exact Sequence . . . . . . . . . . . . . . . . . 116

3. Duality for Finite Group Schemes of Height 1 . . . . . . . . 118

4. Interpretation of Duality in the Category of Sheaves . . . . . 121

5. Duality for $\mu$-Cohomology of surfaces . . . . . . . . . . . . 124

\section{Introduction}

Let $X$ be a smooth proper curve over an algebraically closed field $k$ of characteristic $p$. The purpose of this paper is to extend "Poincare duality" on $X$ to $p$-torsion groups, by allowing as coefficients any finite flat group scheme $A$ on $X$. Let $A^{D}=\operatorname{Hom}_{\text {grp }}\left(A, \mathbb{G}_{m}\right)$ denote the Cartier dual of $A$. Our result (4.9) is that the natural pairing $A \otimes A^{D} \rightarrow \mu_{\infty}$ gives rise to a perfect duality on cohomology.

A new phenomenon which arises when one considers flat coefficients is that their cohomology groups are not, in general, discrete groups. For example, if $X$ is a supersingular elliptic curve, then $H^{1}\left(X, \alpha_{p}\right) \approx H^{1}\left(X, \mathcal{O}_{X}\right)$. This is a $k$-vector space, and its natural algebraic structure should be taken into account.

There are two different contexts in which the duality can be formulated. The first follows a suggestion of Grothendieck that the cohomology theory should take its values in the category of unipotent quasi-algebraic groups. Our original construction was made in this context.

Let $U_{k}$ denote the category of commutative unipotent algebraic groups over $k$, and let $Q U_{k}$ be the corresponding category of quasi-algebraic groups [17]. This is the quotient of the abelian category $U_{k}$ by its subcategory of infinitesimal groups. There is a functor

(0.1) $U^{*} \rightarrow U^{* *}=\underline{R \operatorname{Hom}}\left(U^{*}, \mathbb{Q}_{p} / \mathbb{Z}_{p}\right)$

* Supported by NSF 
on the derived category of $Q U_{k}$, which is an autoduality it has these values:

$$
\begin{aligned}
& \mathbb{Z} / p^{*} \approx \mathbb{Z} / p, \\
& \mathbb{G}_{a}^{*} \approx \mathbb{G}_{a}[1]=\left\{\mathbb{G}_{a}, \text { shifted to degree } 1\right\},
\end{aligned}
$$

or,

$$
\begin{aligned}
& \underline{\operatorname{Hom}}(\mathbb{Z} / p, \mathbb{Q} / \mathbb{Z}) \approx \mathbb{Z} / p, \\
& \underline{\operatorname{Ext}}^{1}(\mathbb{G}, \mathbb{Q} / \mathbb{Z}) \approx \mathbb{G}_{a} .
\end{aligned}
$$

In the first three sections, we define cohomology groups $\underline{H}^{i}(X, A) \in Q U_{k}$ and prove a duality theorem based on (0.1) for group schemes $A$ of height 1, i.e., those on which the Frobenius map $F$ is zero.

Since every unipotent quasi-algebraic group is an extension of a discrete finite group scheme by a connected unipotent group, $\underline{H}^{i}(X, A)$ fits into an exact sequence

$$
0 \rightarrow \underline{U}^{i}(X, A) \rightarrow \underline{H}^{i}(X, A) \rightarrow \underline{D}^{i}(X, A) \rightarrow 0
$$

$\underline{D}^{i}$ being discrete, and $\underline{U}^{i}$ connected. The duality (3.10) implies that
(a) $\underline{D}^{i}(X, A) \approx \underline{\operatorname{Hom}}\left(\underline{D}^{2-i}\left(X, A^{D}\right), \mathbb{Q} / \mathbb{Z}\right)$,
(b) $\underline{U}^{i}(X, A) \approx \underline{\operatorname{Ext}}^{1}\left(\underline{U}^{1-i}\left(X, A^{\mathrm{D}}\right), \mathbb{Q} / \mathbb{Z}\right)$,

so that particular $\underline{D}^{i}=0$, if $i \neq 0,1,2$ and $\underline{U}^{i}=0$ if $i \neq 1,2$.

The second context in which one can formulate duality is in the category of sheaves on the "perfect site" $S_{\text {perf }}, S=$ Spec $k$ (cf. Section 3). To do this we simply replace $\underline{H}^{*}(X, A)$ by $R^{*} \pi, A$, where $\pi: X \rightarrow S$ is the structure map, and take the pairing $R^{*} \pi_{*} A \otimes R^{*} \pi_{*} A^{\mathbb{D}} \rightarrow(\mathbb{Q} / \mathbb{Z})[2]$ induced by cup product. Fortunately, a recent result of Breen [3], (4.1) about the vanishing of certain sheaf Exts on $S_{\text {perf }}$ allows us to do this (Corollary (4.9)).

In both contexts, our method of proof is to resolve by complexes of vector groups, and then to apply Serre duality.

It may be worthwhile to begin by considering the two cases $A=\mu_{p}$ and $A=\alpha_{p}$. There are canonical exact sequences of group schemes
(a) $(0 \longrightarrow \mathbb{Z} / p) \longrightarrow \mathbb{G}_{a} \stackrel{1-\mathbb{F}}{\longrightarrow} \mathbb{G}_{a} \longrightarrow 0$
(b) $\left(0 \longrightarrow \alpha_{p}\right) \longrightarrow \mathbb{G}_{a} \stackrel{-F}{\longrightarrow} \mathbb{G}_{a} \longrightarrow 0$,
(c) $\left(0 \longrightarrow \mu_{p}\right) \longrightarrow \mathbb{G}_{m} \stackrel{F}{\longrightarrow} \mathbb{G}_{m} \longrightarrow 0$,

which we view as resolutions of the left terms. These resolutions can be used to compute the flat cohomology of $\mathbb{Z} / p, \alpha_{p}$, and $\mu_{p}$. Since $\mathbb{G}_{a}, \mathbb{G}_{m}$ are smooth schemes, flat and étale cohomology agree for them [7,11.7], and so we can work with the étale topology on the resolutions.

The first of these sequences is exact for the étale topology, but the other two are not. Instead, the Frobenius map in the étale topology can be continued by differentiation and the Cartier operator [18]. It is known that the following are 
exact sequences of sheaves for the étale topology on a smooth curve $X$ :

$$
\begin{aligned}
& 0 \longrightarrow \mathcal{O}_{X} \stackrel{-F}{\longrightarrow} \mathcal{O}_{X} \stackrel{d}{\longrightarrow} \Omega_{X}^{1} \stackrel{C^{\prime}}{\longrightarrow} \Omega_{X}^{1} \longrightarrow 0, \\
& 0 \longrightarrow \mathcal{O}_{X}^{*} \stackrel{F}{\longrightarrow} \mathcal{O}_{X}^{*} \stackrel{d \log }{\longrightarrow} \Omega_{X}^{1} \stackrel{C^{\prime}-1}{\longrightarrow} \Omega_{X}^{1} \longrightarrow 0,
\end{aligned}
$$

where $C^{\prime}$ denotes the Cartier operator on $\Omega_{X}^{1}$. It follows that the complexes

(a') $0 \longrightarrow \Omega_{X}^{1} \stackrel{c^{\prime}-1}{\longrightarrow} \Omega_{X}^{1} \longrightarrow 0$

(b') $0 \longrightarrow \Omega_{X}^{1} \stackrel{C^{\prime}}{\longrightarrow} \Omega_{X}^{1} \longrightarrow 0$,

can be used to compute cohomology of $\mu_{p}$ and $\alpha_{p}$ respectively, with a shift in dimension. In particular, the sequence ( $\left.\mathrm{a}^{\prime}\right)$ identifies $H^{2}\left(X, \mu_{p}\right)=\mathbb{Z} / p$ as

$$
\operatorname{ker}\left(H^{1}\left(X, \Omega^{1}\right) \stackrel{C^{\prime}-1}{\longrightarrow} H^{1}\left(X, \Omega^{1}\right)\right)=\operatorname{ker}(k \stackrel{F-1}{\longrightarrow} k),
$$

which supplies a fundamental class for duality.

We show (3.4) that the natural pairing $\mathscr{O}_{X} \times \Omega_{X}^{1} \rightarrow \Omega_{X}^{1}$ can be extended to pairings of complexes

(a) $\times\left(a^{\prime}\right) \rightarrow\left(a^{\prime}\right)$,

(b) $\times\left(b^{\prime}\right) \rightarrow\left(a^{\prime}\right)$,

and that these pairings yield the duality for flat cohomology, with coefficients $\left(\mathbb{Z} / p, \mu_{p}\right)$ and $\left(\alpha_{p}, \alpha_{p}\right)$ respectively.

The main part of this paper is the generalization of the complexes (0.5) to arbitrary finite flat group schemes $A$ of height 1 , and of the sequences (0.4) to their Cartier duals $A^{D}$. There are some $p$-th powers in the general form which can not be eliminated, and this changes their appearance slightly.

The sequences $(0.5)$ are related to a Cartier operator for general smooth group schemes which is defined in [4]. Our original construction of the complexes was simplified considerably by the use of ideas of Hoobler [8].

We would like to thank Messing for many helpful conversations we had during the preparation of this paper.

Conventions. All group schemes and sheaves are supposed commutative. There are many formal $p$-th powers which arise, such as the maps $\mathcal{O}_{X} \rightarrow \mathcal{O}_{X^{(p)}}$, or $L \rightarrow L^{(p)}$ if $L$ is an invertible sheaf. Such operations will generally be denoted by a bar: $a \backsim \bar{a}$. This will be unambiguous once the domain and range are specified.

\section{The First Exact Sequence}

Throughout this section $X$ will denote a scheme of characteristic $p$, and $A$ a finite flat group scheme over $X$ of height 1.

Let $e: X \rightarrow A$ be the zero section, let $\mathscr{I} \subset \mathcal{O}_{A}$ be the ideal defining this closed immersion, and let $\operatorname{Inf}_{X}^{1}(A)=\operatorname{Spec}\left(\mathcal{O}_{A} / \mathscr{I}^{2}\right)$ be the first order infinitesimal neighborhood of the zero section. Then $\mathscr{I} / \mathscr{I}^{2}$ is the cotangent space $\omega_{A}$ of $A$ over $X$. Under the above hypothesis it is a locally-free $\mathcal{O}_{X}$-module of finite type [12, II 2.1.4], and hence we may regard it as a vector group over $X$. As such, it represents $\underline{\operatorname{Hom}}_{X \text {-ptd }}\left(\operatorname{Inf}{ }_{X}^{1}(A), \mathbb{G}_{m}\right)$, viewed as a functor on (schemes/X), where the 
notation $X$-ptd means that the morphisms are required to respect the canonical $X$-points of the $X$-schemes $\operatorname{Inf}_{X}^{1}(A)$ and $\mathbb{G}_{m}$. The Cartier dual $A^{D}$ of $A$ represents the functor $\underline{\operatorname{Hom}}_{\mathrm{grp}}\left(A, \mathbb{G}_{m}\right)$ on (schemes/X), and the canonical map from this functor into the first, defined by $\operatorname{Inf}_{X}^{1}(A) \hookrightarrow A$, induces a canonical map

$$
\imath(A): A^{D} \rightarrow \omega_{A} .
$$

The Verschiebung $[5, \mathrm{IV}, 3,4]$ is a map $V_{A}=V: A^{(p)} \rightarrow A$, and it induces a morphism $\omega_{A} \rightarrow \omega_{A^{(p)}}$. Combining this with the canonical isomorphism $\omega_{A^{(p)}}$ $\approx \omega_{A}^{(p)}$, we get a morphism $\omega_{A} \rightarrow \omega_{A}^{(p)}$ which we denote by $\varphi_{0}$. The relative Frobenius for $\omega_{A}$ over $X$ also gives us a morphism $\varphi_{1}: \omega_{A} \rightarrow \omega_{A}^{(p)}$.

(1.1) Proposition. Let $A$ be a finite flat group scheme of height 1 over $X$. The sequence

$$
0 \longrightarrow A^{D} \stackrel{\iota}{\longrightarrow} \omega_{A} \stackrel{\varphi}{\longrightarrow} \omega_{A}^{(p)} \longrightarrow 0,
$$

where $\varphi=\varphi_{0}-\varphi_{1}$, is an exact sequence of group schemes on $X$.

For example, if $A=\mu_{p}$, then $A^{D}=\mathbb{Z} / p$, and $\omega_{A} \approx \omega_{A}^{(p)} \approx \mathbb{G}_{q}$. With these canonical identifications, we have $\varphi_{0}=1, \varphi_{1}=F$. Thus the above sequence becomes the Artin-Schreier sequence $(0.4 \mathrm{a})$. Similarly, the case $A=\alpha_{p}$ reduces to $(0.4 \mathrm{~b})$. So, exactness is clear in these two cases.

We shall need the following lemma.

(1.2) Lemma. Let $0 \rightarrow G^{\prime} \stackrel{i}{\rightarrow} G \stackrel{p}{\rightarrow} G^{\prime \prime} \rightarrow 0$ be a complex of flat group schemes of finite type over a noetherian scheme $X$. Assume that for all geometric points $x$ of $X$, the sequence of fibres $0 \rightarrow G_{x}^{\prime} \rightarrow G_{x} \rightarrow G_{x}^{\prime \prime} \rightarrow 0$ is exact. Then the original sequence is exact.

Proof. The faithful flatness of the $p_{x}$, combined with the local criterion for flatness [6, IV 5.9], imply that $p$ is faithfully flat. Thus $K=\operatorname{Ker}(p)$ is flat and of finite type. The map $i$ factors through $K$. Now the same argument shows that $G^{\prime} \rightarrow K$ is faithfully flat, and hence is an epimorphism. Finally, the kernel of $i$ is a group scheme whose geometric fibres are all trivial, and hence is zero.

As the exactness of the sequence in (1.1) may be checked locally on $X$, and the formation of the sequence commutes with arbitrary changes of base, we may assume, in proving (1.1), that $X$ is noetherian. We check that the hypotheses of Lemma (1.2) hold for our sequence. Note that $t$ is functorial in $A$. On writing out that this functoriality means in the case of the morphism $V_{A}: A^{(p)} \rightarrow A$, we get a commutative diagram,

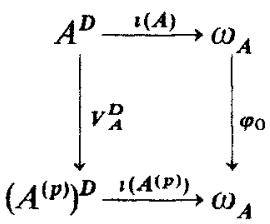


It is known [5, IV , 3, 4.9] that, when $\left(A^{(p)}\right)^{D}$ is identified canonically with $\left(A^{D}\right)^{(p)}$, then $\left(V_{A}\right)^{D}$ becomes identified with $F_{A^{D}}$. Thus $\varphi_{0} \circ \imath(A)=\imath\left(A^{(p)}\right) \circ F_{A^{D}}$.

Also, the functoriality of the relative Frobenius implies that $F_{\omega_{A}} \circ l(A)=$ $l(A)^{(p)} \circ F_{A D}$, and the functoriality of $l$ with respect to $X$ implies that $l(A)^{(p)}=$ $l\left(A^{(p)}\right)$. Thus, $\varphi_{1} \circ l(A)=\imath\left(A^{(p)}\right) \circ F_{A^{D}}$, which shows that $\varphi \circ l(A)=\left(\varphi_{0}-\varphi_{1}\right) l(A)=0$, i.e., that the sequence in question is a complex.

The lemma now reduces us to the case that $X$ is the spectrum of an algebraically closed field. We use induction on the rank of $A$ : If the rank is $p$ then $A \approx \mu_{p}$ or $A \approx \alpha_{p}$, and we have already observed that the proposition holds in these two cases. In general, there will exist an exact sequence $0 \rightarrow A^{\prime} \rightarrow A \rightarrow A^{\prime \prime} \rightarrow 0$ of finite flat group schemes with $A^{\prime}, A^{\prime \prime}$ of smaller rank. Consider the commutative diagram

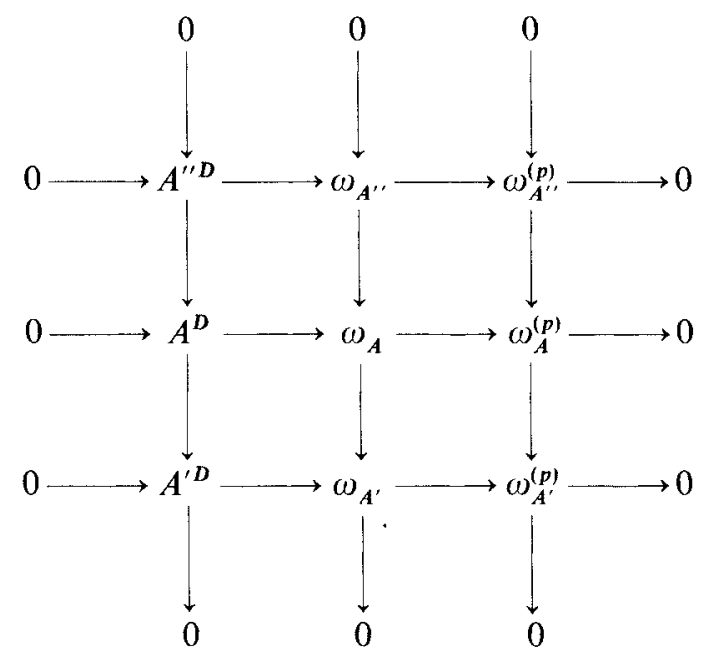

By induction we may assume that the first and third rows are exact. All columns are exact, the first because of the exactness of Cartier duality and the last two because of the exactness of the functor which associates to a group of height one its $p$-Lie-algebra $[5, \mathrm{II}, 7,4.1]$. Since we know the middle row is a complex, it follows that it is also exact.

(1.3) Corollary. Let $\operatorname{cdqc}(X)$ be the cohomological dimension of quasi-coherent sheaves on $X$, and let $A$ be a finite flat group scheme of height 1 on $X$. Then $H^{s}\left(X, A^{D}\right)$ $=0$ if $s>\operatorname{cdqc}(X)+1$.

(1.4) Corollary. Assume, in addition to the above hypotheses, that there is a proper map $\pi: X \rightarrow \operatorname{Spec} k$, with $k$ a field. Then $R^{s} \pi_{*} A^{D}$ is representable by an algebraic group over $k$ for all $s \geqq 0$.

This follows from the exact sequence, since $R^{s} \pi_{*} F$ is represented by a vector group for every coherent sheaf $F$ on $X$. 


\section{The Second Exact Sequence}

Throughout this section, $\pi: X \rightarrow S$ will denote a smooth map of schemes of characteristic $p$, and $S$ will be assumed perfect i.e. such that the absolute Frobenius $S \rightarrow S^{(p)}$ is an isomorphism. We let $X^{\prime}=X^{(1 / p)}$, and denote by $W: X \rightarrow X^{\prime}$ the formal $p$-th power, so that if $F=F_{X^{\prime}}: X^{\prime} \rightarrow X$ is the map defined by the inclusion $\mathcal{O}_{X} \subset \mathcal{O}_{X}^{1 / p}$, then $W F$ is the absolute Frobenius on $X$.

We consider two maps

$$
F_{*} \Omega_{X_{i}^{\prime} S, \text { cl }}^{1} \rightarrow \Omega_{X_{i} S}^{1},
$$

namely, the $\mathscr{O}_{X}$-linear Cartier operator $C$ [9], and the formal $p$-th power map $W^{*}$ defined by $W^{*}(f d x)=\vec{f} d \bar{x}$. (Here the subscript cl denotes closed differentials.)

(2.1) Lemma. There is an exact sequence $C^{\prime}$ of smooth group schemes on $X$ :

$$
C^{*}=0 \rightarrow \mathbb{G}_{m} \stackrel{i}{\longrightarrow} F_{*} \mathbb{G}_{m X^{\prime}} \stackrel{F_{*} d \log }{\longrightarrow} F_{*} \Omega_{X^{\prime}, S, \mathrm{cl}}^{1} \stackrel{C-W^{*}}{\longrightarrow} \Omega_{X_{i} S}^{1} \rightarrow 0 .
$$

Proof. This is standard. See for instance [8].

(2.2) Lemma. Let $A$ be a finite flat group scheme on $X$ of height 1. Let $C^{*}$ denote the sequence of (2.1), and $\mathfrak{a}=\hat{\omega}_{A}$ the Lie algebra of $A$. Then $\operatorname{Hom}\left(A^{D}, C^{*}\right)$ is an exact sequence of group schemes

$$
0 \rightarrow A \stackrel{i}{\longrightarrow} F_{*} A_{X^{\prime}} \stackrel{\partial}{\longrightarrow} \mathfrak{a} \otimes \Omega_{X^{\prime}, S, \mathfrak{c l}}^{1} \stackrel{\Psi}{\longrightarrow} \mathfrak{a} \otimes \Omega_{X / S}^{1} \rightarrow 0 .
$$

The map $\psi$ has the form $\psi=\psi_{0}-\psi_{1}$, with $\psi_{0}=1 \otimes C$ and $\psi_{1}=*^{(p)} \otimes W^{*}, *^{(p)}$ being the $p$-th power operation on the p-Lie algebra a. Thus

$$
\psi(a \otimes \omega)=a \otimes C \omega-a^{(p)} \otimes \bar{\omega} .
$$

Proof. The first two terms can be identified using the functorial property of $F_{*}$, and the last two from the isomorphisms [12, p. 138]

$$
\underline{\operatorname{Hom}}\left(A^{D}, \Omega^{1}\right) \approx \underline{\operatorname{Hom}}\left(\omega_{A}, \mathcal{O}\right) \otimes \Omega^{1} \approx \mathfrak{a} \otimes \Omega^{1}
$$

on $X^{\prime}$ and $X$ respectively. It is clear that the functor $\operatorname{Hom}\left(A^{D},.\right)$ takes the linear map $C$ to $\psi_{0}=1 \otimes C$. On the other hand, $W^{*}$ is linear with respect to the map $F_{*} \mathcal{O}_{X}, \rightarrow \mathcal{O}_{X}$. The $p$-th power map on $\mathcal{O}$ induces the $p$-th power in the Lie algebra. That is, the diagram

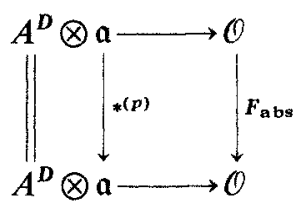

commutes [12, p. 138], or, if $\alpha \in A^{D}$ and $a \in \mathfrak{a}$, then $\overline{\alpha(a)}=\alpha\left(a^{(p)}\right)$. It follows formally that $\operatorname{Hom}\left(A, W^{*}\right)=*^{(p)} \otimes W^{*}$.

Note that $\psi_{0}$ is linear and surjective, whereas $\psi_{1}$ is $F_{\mathrm{abs}}$-linear. It follows that $\psi=\psi_{0}-\psi_{1}$ is a smooth map, and hence is an epimorphism. The fact $[16,6.2 .2]$ that the $f p p f \underline{\operatorname{Ext}}^{1}\left(A^{D}, \mathbb{G}_{m}\right)$ is zero implies that the sequence is exact at the other points. 
(2.3) Lemma. Let $A$ be a finite flat group scheme of height 1 on $X$. Then the map $H^{1}(X, A) \rightarrow H^{1}\left(X^{\prime}, A\right)$ is zero.

Proof. The condition that $A$ has height 1 can be expressed by saying that the $p$-th power map on $\mathcal{O}_{A}$ has its image in $\mathcal{O}_{X} \subset \mathcal{O}_{A}$ :

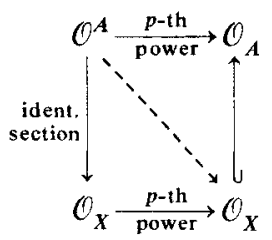

By descent, the same is true for any torsor (homogeneous space) $P$ of $A: \mathcal{O}_{P} \stackrel{\varepsilon}{\longrightarrow} \mathcal{O}_{X}$ $\subset \mathcal{O}_{P}$. The composed map

$$
\mathcal{O}_{P} \stackrel{\varepsilon}{\longrightarrow} \mathcal{O}_{X} \underset{\text { root }}{\stackrel{p-\text { th }}{\longrightarrow}} \mathcal{O}_{X}
$$

is an $\mathcal{O}_{X}$-homomorphism, and gives a point $P$ with values in $X^{\prime}$. Thus the pull-back of $P$ to $X^{\prime}$ is trivial. Since torsors are in one-one correspondence with cohomology classes, the lemma is proved.

(2.4) Proposition. Let $f: X_{\mathrm{fl}} \rightarrow X_{\mathrm{et}}$ denote the morphism of sites induced by the identity map on $X$, where $X_{\mathrm{et}}$ is the small étale site. Let $A$ be a finite flat group scheme of height 1 on $X$. Then $R^{q} f_{*} A=0$ if $q \neq 1$, and there is an exact sequence of sheaves on $X_{\text {et }}$

$$
0 \rightarrow R^{1} f_{*} A \rightarrow \mathfrak{a} \otimes \Omega_{X^{\prime}, \mathrm{s}, \mathrm{cl}}^{1} \stackrel{\psi}{\longrightarrow} \mathfrak{a} \otimes \Omega_{X S}^{1} \rightarrow 0 .
$$

Proof. As is well known, any finite flat group scheme $A$ can be embedded in an exact sequence

$$
0 \rightarrow A \rightarrow G \rightarrow G^{\prime} \rightarrow 0
$$

where $G$ and $G^{\prime}$ are smooth. One can take for $G$ the scheme representing $\mathcal{O}_{A^{D}}^{*}$, as functor on $X$. Since $R^{q} f_{*}$ vanishes on smooth group schemes for $q>0[7]$, it follows that $R^{q} f_{*} A=0$ if $q>1$. Our group $A$ is of height 1 , hence purely infinitesimal, and since $X$ is reduced, $f_{*} A=0$.

Consider the nerve of the covering $X^{\prime} \rightarrow X$ :

$$
X^{\prime \prime \prime} \leftrightharpoons X^{\prime \prime}=X^{\prime} \times_{X} X^{\prime} \longrightarrow X^{\prime} \stackrel{F}{\longrightarrow} X .
$$

Let $A^{\prime}=F_{*} A_{A^{\prime}}$, etc. ... Then this simplicial scheme induces an exact sequence

$$
0 \rightarrow A \rightarrow A^{\prime} \rightarrow A^{\prime \prime} \rightarrow A^{\prime \prime \prime} \rightarrow \cdots
$$

of group schemes on $X[1]$. We consider it a resolution $0 \rightarrow A \rightarrow A^{*}$ of $A$.

The spectral sequence for Cech cohomology [1, V 3.3]

$$
E_{2}^{p q}=\check{H}^{p}\left(X^{\prime} / X, H_{f 1}^{q}(A)\right) \Rightarrow H_{f 1}^{p+q}(X, A),
$$

can be made into a spectral sequence of étale sheaves on $X$ by localization. Then its abutment is $R^{p+q} f_{*} A$. In dimension 1 , the spectral sequence reduces to the 
exact sequence

$$
0 \rightarrow \underline{\breve{H}}_{\mathrm{et}}^{1}\left(X^{\prime} / X, A\right) \stackrel{\bullet}{\longrightarrow} R^{1} f_{*} A \stackrel{u}{\longrightarrow} \underline{\breve{H}}_{\mathrm{et}}^{0}\left(X^{\prime} / X, \underline{H}_{\mathrm{f} 1}^{1}(A)\right)
$$

By Lemma (2.3), every cohomology class on $X$ dies on $X^{\prime}$, and the same is true if $X$ is replaced by any scheme étale over $X$. This implies that the map $u$ is zero, and hence that $v$ is an isomorphism.

The left hand term of (2.5) is kernel modulo image in the sequence $f_{*} A$. Since $X^{\prime}$ is reduced, $f_{*} A^{\prime}=0$. Hence

$$
\underline{\underline{H}}_{\mathrm{et}}\left(X^{\prime} / X, A\right)=\operatorname{ker}\left(f_{*} A^{\prime \prime} \rightarrow f_{*} A^{\prime \prime \prime}\right)=f_{*}\left(\operatorname{ker}\left(A^{\prime \prime} \rightarrow A^{\prime \prime \prime}\right)\right) \text {. }
$$

Let $B=\operatorname{ker}\left(A^{\prime \prime} \rightarrow A^{\prime \prime \prime}\right) \approx A^{\prime} / A$. Then $B$ is a flat group scheme on $X$, and (2.6) $R^{1} f_{*} A=f_{*} B$.

By (2.2)

$$
0 \rightarrow B \rightarrow \mathfrak{a} \otimes \Omega_{X^{\prime}, S, \text { el }}^{1} \stackrel{\psi}{\longrightarrow} \mathfrak{a} \otimes \Omega_{X / S}^{1} \rightarrow 0
$$

is exact, and as we already remarked, $\psi$ is smooth. The proposition now follows by applying $f_{*}$ to this sequence.

(2.7) Remark. The map $R^{1} f_{*} A \rightarrow \mathfrak{a} \otimes \Omega_{X^{\prime} S \text {, cl }}^{1}$ can be described in this way: Let $P$ be a torsor of $A$. Since $A$ is purely infinitesimal, the trivialization $X^{\prime} \stackrel{\varphi^{\prime}}{\longrightarrow} P$ of (2.3) is unique, and gives rise to two maps $X^{\prime} \times X_{X}^{\prime}=X^{\prime \prime} \rightarrow P$, namely $p_{1}^{*} \varphi^{\prime}$ and $p_{2}^{*} \varphi^{\prime}$. Their difference is an element $\alpha^{\prime \prime} \in A\left(X^{\prime \prime}\right)$ which is zero if and only if $\varphi^{\prime}$ is induced by a section of $P$ over $X$. Now if $N$ denotes the nilradical of $\mathcal{O}_{X^{\prime \prime}}$, then $\mathcal{O}_{X^{\prime \prime}} / N \approx \mathcal{O}_{X^{\prime}}$ and $N / N^{2} \approx \Omega_{X^{\prime} / S}^{1}$. Since $\alpha^{\prime \prime}$ must be trivial on $X^{\prime}$, the restriction of $\alpha^{\prime \prime}$ to $\mathscr{O}_{X^{\prime \prime}} / N^{2}$ defines a map $\omega_{A} \rightarrow \Omega_{X^{\prime}, s}^{1}$ i.e., an element of $\mathfrak{a} \otimes \Omega_{X^{\prime}, s}^{1}$.

\section{Duality for Finite Group Schemes of Height 1}

Let $X$ be a smooth projective curve over $S=\operatorname{Spec} k$, where $k$ is a perfect ring. Assume that $X$ has connected, non-empty geometric fibres. In this section, we work with the site $S_{\text {perf }}$, whose objects are perfect schemes, essentially of finite type over $k$, with their étale topology. We also introduce an auxiliary site $X / S_{\text {perf }}$ : Its objects are pairs $\left(U_{1}, S_{1}\right)$ where $S_{1} \in S_{\text {perf }}$ and $U_{1}$ is étale over $X \underset{S}{\times} S_{1}$. A family of maps $\left\{\left(U_{\alpha}, S_{\alpha}\right) \rightarrow\left(U_{1}, S_{1}\right)\right\}$ is a covering in $X / S_{\text {perf }}$ if the maps $U_{\alpha} \rightarrow U_{1}$ and $S_{\alpha} \rightarrow S_{1}$ are étale, and $\left\{U_{\alpha}\right\}$ covers $U_{1}$. If $\pi: X \rightarrow S$ denotes the structure map, we obtain a diagram of sites

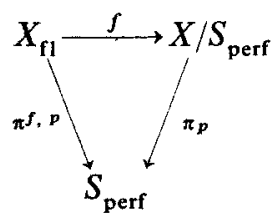


Let $A$ be a finite, flat group scheme of height 1 on $X$. It is clear that Proposition (2.4) extends to the above map $f$. Let us denote the complex

$$
0 \rightarrow \mathfrak{a} \otimes \Omega_{X^{\prime} / S}^{1} \stackrel{\Psi}{\longrightarrow} \mathfrak{a} \otimes \Omega_{X / S}^{1} \rightarrow 0 \rightarrow \cdots
$$

on $X / S_{\text {perf }}$ by $U^{*}=U^{*}(A)$. It follows from Proposition (2.4) that

(3.2) $\mathbb{R} \pi_{f, p^{*}} A \approx \mathbb{R} \pi_{p^{*}} U^{*}[1]$,

the symbol [1] denoting a shift in degree. We denote the complex

$$
0 \rightarrow \omega_{A} \stackrel{\varphi}{\longrightarrow} \omega_{A}^{(p)} \rightarrow 0 \ldots,
$$

which we view as a complex on $X / S_{\text {perf }}$ via $f_{*}$, by $V^{*}=V^{*}\left(A^{D}\right)$. Then

(3.3) $\quad \mathbb{R} \pi_{f, p^{*}} A^{D} \approx \mathbb{R} \pi_{p^{*}} V$.

(3.4) Proposition. The formulas below define a pairing of complexes

$$
\begin{array}{ll}
V^{\prime}\left(A^{D}\right) \times U^{\circ}(A) \rightarrow U^{\prime}\left(\mu_{p}\right): & \\
V^{0} \times U^{0}=\omega_{A} \times \mathfrak{a} \otimes \Omega_{X^{\prime} / S}^{1} \rightarrow \Omega_{X^{\prime} / S}^{1}=U^{0}: & \left(\alpha, a \otimes \omega^{\prime}\right)_{00}=\alpha(a) \omega^{\prime}, \\
V^{1} \times U^{0}=\omega_{A}^{(p)} \times \mathfrak{a} \otimes \Omega_{X^{\prime} / S}^{1} \rightarrow \Omega_{X_{i} S}^{1}=U^{1}: & \left(\beta, a \otimes \omega^{\prime}\right)_{10}=\beta(\bar{a}) \bar{\omega}^{\prime}, \\
V^{0} \times U^{1}=\omega_{A} \times \mathfrak{a} \otimes \Omega_{X_{/ S}}^{1} \rightarrow \Omega_{X_{i} S}^{1}=U^{1}: & (\alpha, a \otimes \omega)_{01}=\alpha(a) \omega .
\end{array}
$$

Proof. A pairing is a map $V^{*} \otimes_{\mathbf{z}} U^{\cdot} \rightarrow U^{*}\left(\mu_{p}\right)$, and so the given maps are the required data. We must show that they define a map of complexes. This means we have to check that

$$
\psi_{\mu}\left(\alpha, a \otimes \omega^{\prime}\right)_{00}=\left(\varphi(\alpha), a \otimes \omega^{\prime}\right)_{10}+\left(\alpha, \psi\left(a \otimes \omega^{\prime}\right)\right)_{01} .
$$

Expanding the right side by (1.1) and (2.2), we get

$$
\begin{gathered}
\left(\varphi_{0} \alpha, a \otimes \omega^{\prime}\right)_{10}-\left(\bar{\alpha}, a \otimes \omega^{\prime}\right)_{10}+\left(\alpha, a \otimes C \omega^{\prime}\right)_{01}-\left(\alpha, a^{(p)} \otimes \bar{\omega}^{\prime}\right)_{01} \\
=\varphi_{0} \alpha(\bar{a}) \bar{\omega}^{\prime}-\bar{\alpha}(\bar{a}) \bar{\omega}^{\prime}+\alpha(a) C \omega^{\prime}-\alpha\left(a^{(p)}\right) \bar{\omega}^{\prime} .
\end{gathered}
$$

The left side is

$$
C\left(\alpha(a) \omega^{\prime}\right)-\overline{\alpha(a) \omega^{\prime}}=\alpha(a) C \omega^{\prime}-\bar{\alpha}(\bar{a}) \bar{\omega}^{\prime} .
$$

Thus we need only verify the equality

or

$$
\varphi_{0} \alpha(\bar{a}) \bar{\omega}^{\prime}=\alpha\left(a^{(p)}\right) \bar{\omega}^{\prime}
$$

$$
\varphi_{0} \alpha(\bar{a})=\alpha\left(a^{(p)}\right)
$$

Consider the two maps $\omega_{A} \rightarrow \underline{\operatorname{Hom}}\left(\mathbf{a}, \mathbb{G}_{a}\right)$

$$
\begin{aligned}
& \alpha \mapsto\left(a \mapsto \alpha\left(a^{(p)}\right)\right) \\
& \alpha \mapsto\left(a \mapsto \varphi_{0}(\alpha)(\bar{a})\right) .
\end{aligned}
$$

Since these are both linear, they will be equal if they agree on the subgroup $\imath A^{D} \subset \omega_{A}\left(l: A^{D} \rightarrow \omega_{A}\right.$ is universal for maps into vector groups [10,1.4]). If $\alpha$ arises from an $\alpha_{0}$ in $A^{D}$ then (1.1) $\varphi_{0}(\alpha)=\varphi_{1}(\alpha), \varphi_{1}(\alpha)(\bar{a})=\left(F_{A^{D}} \alpha_{0}\right)(\tilde{a})=\bar{a}\left(F_{A^{D}} \alpha_{0}\right)$ 
(regarding $a$ as an element of $\underline{\operatorname{Hom}}\left(A^{D}, \mathbb{G}_{a}\right)=\mathfrak{a}$ ), and $\bar{a}\left(F_{A^{D}} \alpha_{0}\right)=\left(F_{\mathbb{G}_{0}} \circ a\right)\left(\alpha_{0}\right)=$ $\left(a^{(p)}\right)\left(\alpha_{0}\right)=\alpha\left(a^{(p)}\right)[15$, p. 138].

Since they are functorial, the definitions of $U^{\prime}, V^{*}$ and the pairing extend to any bounded complex $A^{\circ}$ of groups of height 1 . The terms appearing in the complexes $U^{*}, V^{*}$ are all locally free coherent $\mathcal{O}_{X}$-modules, and so we can use Čech cohomology for some affine open cover of $X$ to compute $\mathbb{R} \pi_{p^{*}}$ for them. We then obtain Cech complexes $\breve{C}^{*}\left(U^{*}\right), \breve{C}^{*}\left(V^{*}\right)$, each of whose terms is represented as a quasi-coherent $\mathcal{O}_{s}$-module, but whose maps are not $\mathcal{O}_{s}$-linear. Using cup product on Cech cocycles, we can deduce from the pairing (3.4) a pairing

$$
\check{C}^{\cdot}\left(U^{\cdot}\left(A^{*}\right)\right) \times \check{C}^{\cdot}\left(V^{*}\left(A^{\cdot D}\right)\right) \rightarrow \check{C}^{\cdot}\left(U^{*}\left(\mu_{p}\right)\right) .
$$

We have

$$
U^{*}\left(\mu_{p}\right)=\left[\Omega_{X^{\prime}}^{1} \stackrel{C-F}{\longrightarrow} \Omega_{X}^{1}\right]
$$

and so the trace map $H^{1}\left(X, \Omega_{X}^{1}\right) \stackrel{\sim}{\longrightarrow} \mathbb{G}_{a}$ induces a map of complexes

$$
\check{C} \cdot\left(U^{\cdot}\left(\mu_{p}\right)\right) \stackrel{\operatorname{tr}}{\longrightarrow}\left[\mathbb{G}_{a} \stackrel{1-F}{\longrightarrow} \mathbb{G}_{a}\right] .
$$

Now it is easily seen that one can choose a covering so that the complexes $\check{C}^{\text {* }}$ are limits of quasi-isomorphic subcomplexes each of whose terms is a locally free $\mathcal{O}_{s}$-module. For instance, we can cover $X$ by two affine opens of the from $X-D, X-D^{\prime}$, where $D$ and $D^{\prime}$ are disjoint Cartier divisors. Then the Čech complex for any coherent sheaf $F$ is the limit of the acyclic complexes

$$
0 \rightarrow \Gamma\left(X, F(n D) \oplus F\left(n D^{\prime}\right)\right) \rightarrow \Gamma\left(X, F\left(n D+n D^{\prime}\right)\right) \rightarrow 0 \quad(n \gg 0) .
$$

So, the $\breve{C}^{\cdot}$ define canonical elements in the derived category $D^{b}\left(Q U_{k}(p)\right)$, where $Q U_{k}$ is the category of quasi-algebraic unipotent group schemes over $k$, and the symbol $(p)$ denotes the subcategory of elements annihilated by $p$. Let us denote these elements by

$$
\begin{aligned}
& \mathbb{H}^{*}\left(X, A^{*}\right)=\check{C}^{*}\left(U^{*}\left(A^{*}\right)\right)[1], \\
& \mathbb{H}^{(}\left(X, A^{\cdot D}\right)=\check{C}^{*}\left(V^{*}\left(A^{*}\right)\right) .
\end{aligned}
$$

Suppose now that $k$ is a perfect field. Then we also write $\underline{H}^{q}(X, A)$ and $\underline{H}^{q}\left(X, A^{\cdot D}\right)$ for the cohomology of the above complexes. These are the elements of $Q U_{k}(p)$ which represent the functors $R^{q} \pi_{f, p^{*}} A^{*}$ and $R^{q} \pi_{f, p^{*}} A^{{ }^{D}}$ on $S_{\text {perf }}$. In particular, if $k$ is algebraically closed, then we have

$$
\begin{aligned}
& H^{q}\left(X, A^{*}\right)=\Gamma\left(S, \underline{H}^{q}\left(X, A^{*}\right)\right) \\
& H^{q}\left(X, A^{\cdot D}\right)=\Gamma\left(S, \underline{H}^{q}\left(X, A^{\cdot D}\right)\right) .
\end{aligned}
$$

The group $\mathbb{G}_{a}$ is an injective object in $Q U_{k}(p)$, and so $\left[\mathbb{G}_{a} \stackrel{1-F}{\longrightarrow} \mathbb{G}_{a}\right]$ is a resolution of $\mathbb{Z} / p$. Therefore the pairing (3.4) and the trace map (3.6) define a map

$$
\mathbb{H}^{*}\left(X, A^{*}\right) \rightarrow \mathbb{R} \underline{\operatorname{Hom}}_{Q U_{k}(p)}\left(\mathbb{H}^{*}\left(X, A^{\cdot}\right), \mathbb{Z} / p\right)[2] .
$$

On the other hand, the complex $\mathbb{H}^{\circ}\left(X, A^{\circ}\right)$ is made up of finite-dimensional $k$-vector spaces, and if $V$ is any such vector space, then $\operatorname{Ext}_{Q U_{k}(p)}^{i}(V, \mathbb{Z} / p)=V^{\vee}=$ $\operatorname{Hom}_{\mathrm{v} \text {.sp. }}(V, k)$ if $i=1$, and is zero otherwise. The isomorphism of $\operatorname{Ext}^{1}$ with the 
linear dual $V^{\vee}$ is given by using the resolution $\left[\mathbb{G}_{a} \stackrel{1-F}{\longrightarrow} \mathbb{G}_{a}\right]$ and identifying $V^{\vee}$ with the subgroup of $\operatorname{Hom}_{Q U_{k}(p)}\left(V, \mathbb{G}_{a}\right)$ of linear maps. Therefore we have a quasiisomorphism

$$
R \operatorname{Hom}_{Q v_{k}(p)}\left(\mathbb{H}^{*}\left(X, A^{\cdot D}\right), \mathbb{Z} / p\right) \rightarrow \mathbb{H}^{*}\left(X, A^{\cdot D}\right)^{\vee}[1] .
$$

(3.10) Theorem. Let $k$ be a perfect field. Then the map (3.8) is an isomorphism.

Proof. We may assume that $A^{*}$ has only one non-zero term. Then we have to show that the map induced from (3.9):

$$
\underline{H}^{i}(X, A) \rightarrow H^{i}\left(\mathrm{H}^{*}\left(X, A^{D}\right)^{\vee}\right)[1]
$$

is an isomorphism, for all $i$. We go back to the complexes $U^{*}(A), V^{*}\left(A^{D}\right)$. They give rise to distinguished triangles

$$
\mathbb{H}^{\prime}(X, A)[-1] \rightarrow \mathbb{H}^{\cdot}\left(X, U^{0}\right) \rightarrow \mathbb{H}^{\cdot}\left(X, U^{1}\right)
$$

and

$$
\mathbb{H}^{*}\left(X, A^{D}\right) \rightarrow \mathbb{H}^{*}\left(X, V^{0}\right) \rightarrow \mathbb{H}^{*}\left(X, V^{1}\right) .
$$

The pairing (3.4) $V^{0} \times U^{1} \rightarrow \Omega_{X}^{1}$ is the pairing of Serre duality. So is $V^{1} \times U^{0} \rightarrow \Omega_{X}^{1}$, if we identify $\mathfrak{a} \otimes \Omega_{X}^{1}$, with $\mathbf{a}^{(p)} \otimes \Omega_{X}^{1}$ by the map $\alpha \otimes \omega^{\prime} \rightsquigarrow \bar{\alpha} \otimes \bar{\omega}^{\prime}$. Using these pairings, the maps (3.11) fit into a long exact sequence, and the theorem follows from Serre duality and the 5-lemma.

\section{Interpretation of Duality in the Category of Sheaves}

Using a recent theorem of Breen, we can generalize the results of the previous section, by dualizing in the category of sheaves. Let $S$ be a perfect scheme, and let $S_{\text {perf }}$ be the site on $S$ introduced in the previous section. The result we use is

(4.1) Theorem (Breen [2]). For all $i>0, \underline{\operatorname{Ext}}^{i}\left(\mathbb{G}_{a}, \mathbb{G}_{a}\right)=0$, where Ext denotes extensions in the category of sheaves of $\mathbb{Z} / p$-modules on $S_{\text {perf }}$.

It follows from the Artin-Schreier sequence

$$
0 \rightarrow \mathbb{Z} / p \rightarrow \mathbb{G}_{a} \stackrel{1-F}{\longrightarrow} \mathbb{G}_{a} \rightarrow 0
$$

that for any complex $L$ of locally free sheaves, the linear dual $\operatorname{Hom}_{\mathscr{O}}(L, \mathcal{O})$ is

(4.2) $L=\underline{R \operatorname{Hom}_{\mathbb{Z} / p}}(L, \mathbb{Z} / p)[-1]$,

in the derived category of sheaves on $S_{\text {perf }}$ (cf. [14]).

Let $\pi: X \rightarrow S$ be a smooth projective curve with connected, non-empty geometric fibres, as in Section 3, and let $A^{\prime}$ be a complex of finite group schemes on $X$ of height 1 . As was seen in Section $3, R \pi_{*} A^{*}$ and $R \pi_{*} A^{* D}$ can be represented by complexes $\mathbb{H}^{*}(3.7)$ of locally free $\mathcal{O}_{\mathrm{S}}$-modules, if $S$ is affine. Hence the formula (4.2) can be applied.

The pairing $A^{*} \otimes A^{D} \rightarrow \mu_{p}$ induces by cup product a pairing

$$
R \pi_{*} A^{*} \otimes R \pi_{*} A^{\cdot D} \rightarrow R \pi_{*} \mu_{p}
$$


and since $R^{2} \pi_{*} \mu_{p}=\mathbb{Z} / p$ and $R^{q} \pi_{*} \mu_{p}=0$ if $q>2$, there is a map in the derived category:

(4.4) $R \pi_{*} \mu_{p} \rightarrow(\mathbb{Z} / p)[2]$.

As in the previous section, Serre duality and the 5-lemma imply

(4.5) Theorem. Let $S$ be a perfect scheme, and $X \stackrel{\pi}{\longrightarrow} S$ a smooth projective curve with connected, non-empty geometric fibres. Let $A^{\circ}$ be a complex of finite flat groups of height 1 on $X$. Then the cup product pairing

$$
R \pi_{\mu} A^{\cdot} \otimes R \pi_{*} A^{\cdot D} \rightarrow(\mathbb{Z} / p)[2]
$$

is a perfect duality on $S_{\text {perf }}$, i.e. the induced map

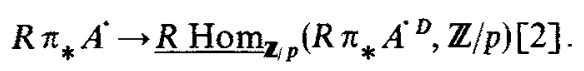

is an isomorphism, where $\underline{R}$ Hom is taken in the derived category of sheaves on $S_{\text {perf }}$.

Proof. The only point which has to be checked in order to apply Serre duality, is that the explicit formulas (3.4) agree with the cup product (4.3). Now the formulas (3.4) on $X / S_{\text {perf }}$ were used to define a pairing by cup product on Cech cochains, and it is known that the Cech cup product agrees with the cup product induced from injective resolutions. So, it suffices to show that they agree with cup product for the map $f: X_{\mathrm{f} 1} \rightarrow X / S_{\text {perf }}$ of diagram (3.1). In other words, we have to prove.

(4.6) Lemma. The diagram

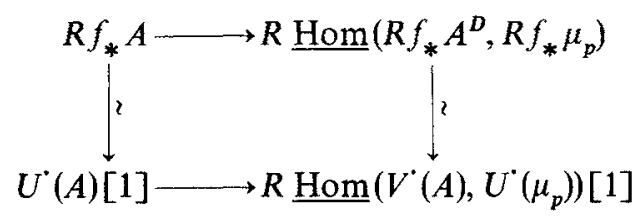

commutes, where the top map is induced "by cup product" from the pairing $A \otimes A^{D}$ $\rightarrow \mu_{p}$, and the bottom arrow is defined by (3.4).

Proof. Let $0 \rightarrow A \rightarrow Z^{*}(A)$ denote the resolution (2.2), and consider the diagram of pairings on $X_{\mathrm{fl}}$ :

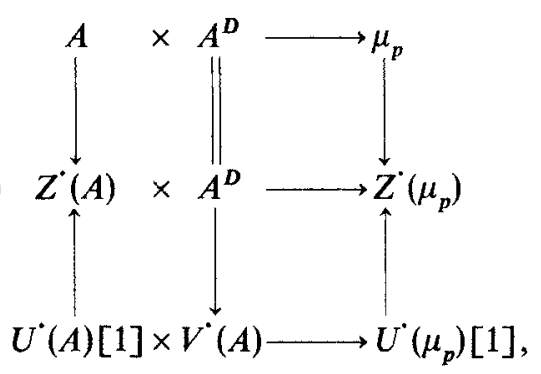

where the middle pairing is determined by Lemma (2.2), and the bottom one by (3.4). We will check that this diagram commutes, from which it will follow that the 
induced diagram

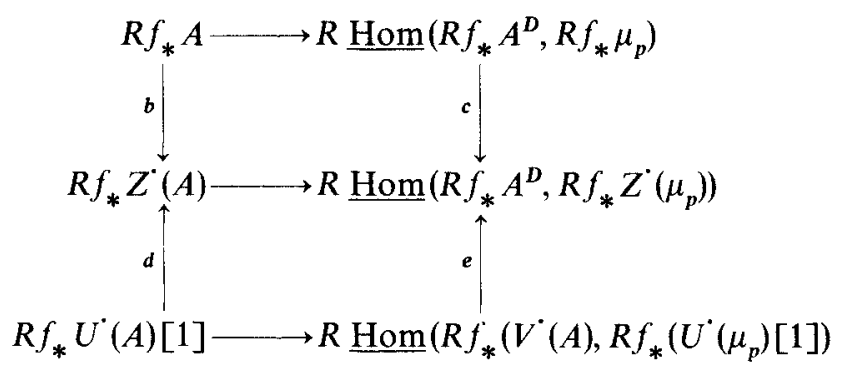

also commutes. Since $A \rightarrow Z^{\prime}(A)$ is a quasi-isomorphism for all $A$, the maps $b, c$ are quasi-isomorphisms. Moreover, since $U^{*}(A)$ and $V^{*}(A)$ are complexes of smooth groups, $R f_{*} U^{\prime}(A)=f_{*} U^{*}(A)$, which we denote by $U^{*}(A)$ on $X / S_{\text {perf }}$, etc. .... It follows from (1.1) and (2.4) that the maps $d, e$ are also quasi-isomorphisms, and the lemma follows.

The commutativity of the upper part of (4.7) is just Lemma (2.2). The lower part, when decomposed, leads to two diagrams which have to be checked:

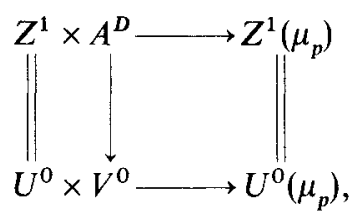

and

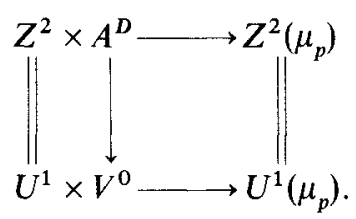

Both verifications are the same. The bottom square expands to

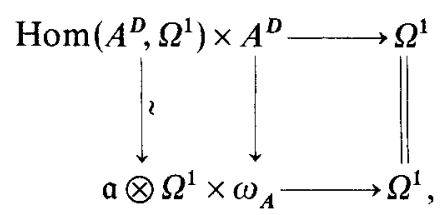

where the pairings are the obvious ones, and the left vertical arrow is the isomorphism [15] used in (2.2). It is clear that this commutes.

Suppose now that $S=\operatorname{Spec} k$, where $k$ is a perfect field. Then any finite flat group scheme $A$ on $X$ which is $p$-torsion has a filtration whose successive quotients are either of height 1 , or have Cartier duals of height 1 . It can be shown by formal arguments [14] that

$R \underline{\operatorname{Hom}}_{\mathbf{Z}}(\mathscr{F}, \mathbb{Z} / p) \approx R \underline{\operatorname{Hom}}_{\mathbf{Z}}(\mathscr{F}, \mathbb{Q} / \mathbb{Z})$ 
for any sheaf of $\mathbb{Z} / p$-modules $\mathscr{F}$ on $S_{\text {perf }}$. Therefore the above duality (4.5) can be restated by saying that the canonical map

$$
R \pi_{*} A^{*} \rightarrow \underline{R \operatorname{Hom}_{\mathbf{Z}}}\left(R \pi_{*} A^{\cdot D}, \mathbb{Q} / \mathbb{Z}\right)[2]
$$

defined by the pairing $A^{*} \otimes A^{D} \rightarrow \mu_{\infty}$, is an isomorphism when $A$ has height 1 , and the previous statement allows this to be extended to all finite groups.

(4.9) Corollary. Suppose $S=\operatorname{Spec} k$ where $k$ is a perfect field, and let $A$ be any complex of finite flat groups schemes on $X$. Then cup product induces a perfect duality

$$
R \pi_{*} A^{*} \times R \pi_{*} A^{D} \rightarrow \mathbb{Q} / \mathbb{Z}[2],
$$

in the category of sheaves on $S_{\text {perf }}$.

\section{Duality for $\mu$-Cohomology of Surfaces}

In this section $X \stackrel{\pi}{\longrightarrow} S$ will denote a smooth proper algebraic surface over $S=$ Spec $k$, where $k$ is a perfect field. As an application of our results we will describe a "Poincaré duality" for $\mu$-cohomology on $X$, i.e., an isomorphism

(5.1) $R \pi_{*} \mu_{p^{v}} \stackrel{\sim}{\longrightarrow} \underline{\operatorname{Hom}}\left(R \pi_{*} \mu_{p^{v}}, \mathbb{Q} / \mathbb{Z}\right)[4]$

in the derived category of sheaves on $S_{\text {perf }}$. The pairing will be described in terms of a Lefschetz pencil on $X$ and is based on ideas developed in joint work with Mazur on arithmetic surfaces. For an invariant treatment of flat duality on surfaces, we refer the reader to [14].

A Lefschetz pencil on $X$ gives rise [10] to a diagram

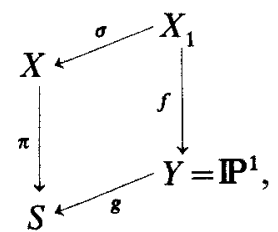

where $\sigma$ is the blowing-up of the base points of the pencil, and where $f$ is smooth except on a finite set of points, at which the map has an ordinary singularity and the fibre has a single node. Let $\Delta$ denote the finite set of points of $Y$ above which $f$ is singular. We will work with the scheme $X_{1}$, and will omit the proof that the duality of $X_{1}$ induces one on $X$. Let us therefore replace $X$ by $X_{1}$.

The duality will be described as a cup product arising from a natural pairing

$$
R f_{*} \mu_{p^{v}} \otimes R f_{*} \mu_{p^{v}} \rightarrow \mu_{p^{v}}
$$

of sheaves on $Y$. We can compute $R f_{*} \mu_{p^{\nu}}$ from $R f_{*} \mathbb{G}_{m}$. The relevant values are

$$
\begin{aligned}
& f_{*} \mathbb{G}_{m}=\mathbb{G}_{m} \\
& R^{1} f_{*} \mathbb{G}_{m}=\underline{\operatorname{Pic}} X / Y=J \otimes \mathbb{Z},
\end{aligned}
$$


where $J$ is the family of generalized jacobians of $X / Y$, and $\mathbb{Z}$ measures degree of a line bundle on a fibre. This group $\mathbb{Z}$ is split off using a chosen section $s: Y \rightarrow X$. It is known that $R^{q} f_{*} \mathbb{G}_{m}$ is uniquely divisible if $q \geqq 2$, for any proper map $X \stackrel{f}{\longrightarrow} Y$ of relative dimension $\leqq 1$. Therefore, multiplication by $p^{v}$ in (5.3) shows that

$$
R^{a} f_{*} \mu_{p^{v}}= \begin{cases}\mu_{p^{v}} & q=0 \\ P_{v} & q=1 \\ \mathbb{Z} / p^{v} & q=2\end{cases}
$$

where $P_{v}$ denotes the kernel of multiplication by $p^{v}$ in $J$. Moreover, the sheaves $\mu_{p v}$ and $\mathbb{Z} / p^{v}$ can be split off of this complex by using the section, i.e.,

$$
R f_{*} \mu_{p^{v}} \approx \mu_{p^{v}} \oplus P_{v}[1] \oplus \mathbb{Z} / p[2]
$$

The main part of our discussion will be to show that the natural pairing $P_{v} \otimes P_{v} \rightarrow \mu_{p^{v}}$ induces by cup product a perfect duality

$$
R g_{*} P_{v} \otimes R g_{*} P_{v} \rightarrow \mathbb{Q} / \mathbb{Z}[2] .
$$

Since $R g_{*} \mu_{p^{v}}$ and $R g_{*} \mathbb{Z} / p^{v}$ are also dual (4.9), this will provide the duality

$$
R \pi_{*} \mu_{p^{v}} \otimes R \pi_{*} \mu_{p^{v}} \rightarrow \mathbb{Q} / \mathbb{Z}[4]
$$

by Lemma (5.5). Unfortunately the sheaf $P_{v}$ is a quasifinite, flat group scheme on $Y$ which is not finite above the set $\Delta$, and so the results of Section 4 can not be applied directly.

Let us recall the theory of autoduality of the jacobian for Néron models. (This theory is not yet completely published, but see $[2,12]$.) Let $Z \stackrel{f}{\longrightarrow} Y$ be any proper map of a smooth surface $Z$ to a smooth curve $Y$, such that the geometric fibres are connected and the general fibre is smooth. Assume that $f$ has a section. Let $J$ denote the Néron model of the jacobian of the general fibre, and let $J^{0}$ be the open subgroup whose fibres are the connected components of the fibres of $J$. Then $J / J^{0}=\varepsilon$ is an étale skyscraper sheaf which is concentrated at the points of $Y$ above which the fibre of $f$ is reducible. The autoduality on the small smooth site $Y_{\text {smooth }}$ is a pair of natural isomorphisms

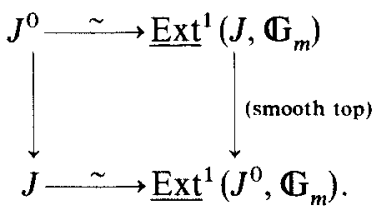

Moreover, the maps (5.7) induce an isomorphism

$$
\varepsilon \stackrel{\sim}{\longrightarrow} \operatorname{Ext}^{2}\left(\varepsilon, \mathbb{G}_{m}\right) . \quad \text { (smooth top) }
$$

If we pass to the flat topology, the maps $(5.7,8)$ are of course still defined, but they are no longer isomorphisms.

There are no reducible fibres for our map $X \stackrel{f}{\longrightarrow}, Y$. Hence $J=J^{0}$. Multiplication by $p^{v}$ in the map (5.7) induces the required pairing

$$
P_{v} \otimes P_{v} \rightarrow \mu_{p^{v}}
$$


and to show that cup product via this pairing induces an autoduality, it is enough to treat the case $v=1$. Let us write $P$ for $P_{1}$, and $\mu$ for $\mu_{p}$.

We need to consider a second surface fibred over $Y$, namely the minimal regular model $\bar{X}$ for the fibration $Y \times_{Y^{(p)}} X^{(p)}=V$ over $Y$. The map $f^{(p)}: X^{(p)} \rightarrow Y^{(p)}$ is a Lefschetz fibration like $f$, and so $V$ is smooth except above the points $y \in A$. A local analysis at the singular points of $f$ shows that $V$ has a single $A_{p-1}$ singularity [11] above $y \in \Delta$. Let $\bar{f}: \bar{X} \rightarrow Y$ denote the resolution of the singularities of $V$. Each singular fibre of $\bar{f}$ has a graph of the form

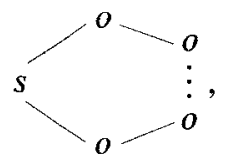

where there are $p-1$ vertices $o$ corresponding to rational curves, and $s$ corresponds to a curve of genus $g-1$, the normalization of the nodal curve $X_{y}^{(p)}$. All components have self-intersection -2 .

Let $\bar{J}$ denote the Néron model on $Y$ of the jacobian of the general fibre of $\bar{f}$. The form of the degenerations (5.10) shows [2] that $\varepsilon=\vec{J} / \bar{J}^{0}$ is a skyscraper sheaf having stalk $\mathbb{Z} / p$ at each point of $\Delta$ :

$$
\varepsilon=\bigoplus_{y \in \Delta}(\mathbb{Z} / p)_{y} .
$$

Now the fibres of $V / Y$ are the $p$-th powers of the fibres of $f$ :

$X_{y}^{(p)}=V_{y}$.

Also, of course, the jacobian of $X_{y}^{(p)}$ is $J_{y}^{(p)}$. Therefore the relative Picard scheme Pic $^{0} V / Y$ is $Y \times_{Y(p)} J^{(p)}$. On the other hand, we may relate $\underline{\mathrm{Pic}}^{0} V / Y$ to $\bar{J}$ via the resolution $\bar{X} \rightarrow V$, and this shows $\underline{\operatorname{Pic}}^{0} V / Y=J^{0}$. Thus $\bar{J}^{0}=Y \times_{Y^{(p)}} J^{(p)}$, and so the relative Frobenius defines a map $J \stackrel{F}{\longrightarrow} \bar{J}^{0}$. Let $A$ denote its kernel, which is a finite flat subgroup of $J$ of $p$-rank $g$.

(5.12) Lemma. The above groups form a row-exact diagram

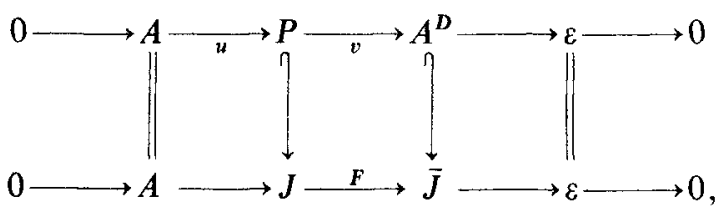

where the top row is self-dual in the sense that the diagram of pairings

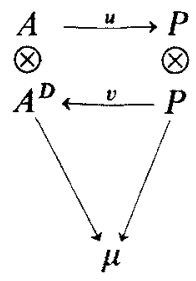

commutes. 
We defer the proof of the lemma, and proceed to show that (5.6) is a duality. Let $\dot{L}$ denote the complex $0 \rightarrow A \rightarrow P \rightarrow A^{D} \rightarrow 0$, which we view as a resolution of $\varepsilon$. The autoduality of $L$ defines a map

$$
\dot{L}[2] \rightarrow \underline{R ~ H o m}_{\mathbb{Z} / p}(L, \mu),
$$

and hence by cup product a map

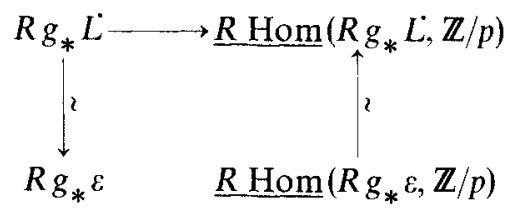

in the derived category of sheaves of $\mathbb{Z} / p$-modules on $S_{\text {perf }}$.

It is enough to prove that this map is an isomorphism. For, we know by (4.9) that $R g_{*} A$ and $R g_{*} A^{D}$ are dual, and so it will follow that

$$
R g_{*} P \stackrel{\sim}{\longrightarrow} \operatorname{Hom}\left(R g_{*} P, \mathbb{Z} / p\right),
$$

as required.

Since $\varepsilon$ is an étale skyscraper sheaf (5.11), we have

$$
R g_{*} \varepsilon=g_{*} \varepsilon=\underset{y \in \Delta}{\bigoplus} \mathbb{Z} / p
$$

What has to be checked is that the obvious pairing on this group is induced by (5.13). The fundamental class is in $H^{2}(Y, \mu)=\operatorname{Ext}^{2}(\mathbb{Z} / p, \mu)$, hence we can interpret the image of the cup product map (5.13) as

$$
\operatorname{Ext}^{2}(\varepsilon, \mu) \stackrel{\sim}{\longrightarrow} \operatorname{Hom}\left(g_{*} \varepsilon, \mathbb{Z} / p\right),
$$

where these are viewed as sheaves on $S_{\text {perf }}$. Let us replace $\operatorname{Ext}_{\mathbf{z} p}^{2}(\varepsilon, \mu)$ by the isomorphic sheaf $\operatorname{Ext}_{\mathbf{Z}}^{2}\left(\varepsilon, \mathbb{G}_{m}\right)$, and work with extensions of $\mathbb{Z}$-modules. We may compute $\operatorname{Ext}^{2}\left(\varepsilon, \mathbb{G}_{m}\right)$ locally at the points $y \in \Delta$ since $\varepsilon$ is a skyscraper sheaf. Choose such a point $y$. Then the map (5.13) can be described this way: Let $\alpha \in A^{D}$ be a local section which maps to the canonical generator $t$ of $\varepsilon_{y}$. Then the upper exact sequence of (5.12) may be pushed out via $\alpha$ to obtain an element $e \in$ $\operatorname{Ext}^{2}\left(\varepsilon, \mathbb{G}_{m}\right)$. This is the image of $t$. Thus we have to show that $e$ generates $\operatorname{Ext}^{2}\left(\varepsilon, \mathbb{G}_{m}\right)$, locally at $y$. We pass to the lower sequence of (5.12), in which $\varepsilon_{y}$ is interpreted as the group of connected components of the fibre $\bar{J}_{y}$, and so we view $\alpha$ as a section of $\bar{J}$ which passes through the component of $\bar{J}_{y}$, corresponding to $\imath$. By (5.7), $\bar{J}$ maps to $\operatorname{Ext}^{1}\left(\bar{J}^{0}, \mathbb{G}_{m}\right)$, and the extension corresponding to $\alpha$ is of course obtained by push-out from the sequence

$$
0 \rightarrow A \rightarrow J \rightarrow \bar{J}^{0} \rightarrow 0 \text {. }
$$

The fact that $e$ generates $\operatorname{Ext}^{2}\left(\varepsilon, \mathbb{G}_{m}\right)$ now follows from (5.8). 
(5.12) Proof of Lemma. The lower sequence is exact by construction. Consider the diagram

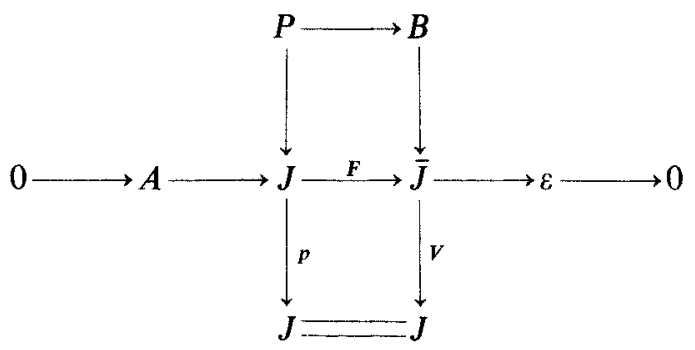

where $V$ is the extension of Verschiebung on the general fibre, which exists by the Néron property of $J$, and where $B=\operatorname{ker} V$. Since the columns are exact when zeros are added at both ends, the serpent lemma defines an exact sequence

$$
0 \rightarrow A \rightarrow P \rightarrow B \rightarrow \varepsilon \rightarrow 0,
$$

and it remains to show that $B=A^{D}$. Presumably, this fact follows from the duality (5.7) applied to the maps $F: J \rightarrow \bar{J}^{0}$ and $V: \bar{J} \rightarrow J$. In any case, (5.7) defines a map $B \rightarrow A^{D}=\underline{\operatorname{Hom}}\left(A, \mathbb{G}_{m}\right)$. Moreover this map is an isomorphism at all points at which $J$ and $\bar{J}$ are abelian schemes, i.e., except possibly at $y \in \Delta$. It is easily checked that $B$ is a finite, flat group scheme. Therefore it suffices (1.2) to show that the map of fibres $B_{y} \rightarrow A_{y}^{D}$ is an isomorphism when $y \in \Delta$. Now the group scheme $J_{y}$ is an extension of an abelian variety $J_{y}^{\prime}$ of dimension $g-1$ by $\mathbb{G}_{m}$, and so is $\bar{J}_{y}^{0}$. We have a diagram

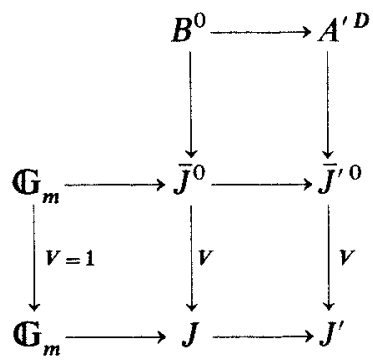

where all terms are to be supplied with subscripts $y, B^{0}=B \cap \bar{J}^{0}$, and $A^{\prime}$ is the kernel of $F$ on $J^{\prime}$. Therefore

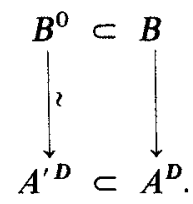

The cokernels of both inclusions are $\mathbb{Z} / p$. Hence $B \approx A^{D}$. This completes the proof. 


\section{References}

1. Artin, M., Grothendieck, A., Verdier, J.-L.: Cohomologie étale des schémas II. Lecture Notes in Math. 270. Berlin-Heidelberg-New York: Springer 1972

2. Artin, M. (Notes by Wyman, B.): Autoduality of the Jacobian. Bowdoin College 1967 (mimeographed notes)

3. Breen, L.: In preparation

4. Cartier, P.: Une nouvelle opération sur les formes différentielles. C.R. Acad. Sci. Paris 244, 426-428 (1957)

Calcul differential sur les variétés algébriques en caractéristique non nulle. loc. cit. 245, 1109-1111 (1957)

5. Demazure, M., Gabriel, P.: Groupes algébriques, Tome 1. Amsterdam: North-Holland 1970

6. Grothendieck, A.: Revêtements étales et group fondamental. Lecture Notes in Math. 224. BerlinHeidelberg-New York: Springer 1971

7. Grothendieck, A.: Le groupe de Brauer III, appendix. Dix exposés sur la cohomologie des schémas. Amsterdam: North-Holland 1968

8. Hoobler, R.: Cohomology of purely inseparable Galois coverings. J. Reine u. Angew. Math. 266, 183-199 (1974)

9. Katz, N.: Nilpotent connections and the monodromy theorem: applications of a result of Turritin. Publ. Math. I.H.E.S. 39, 175-232 (1971)

10. Katz, N.: Groupes de Monodromie en géométrie algébrique, exposé XVII. Lecture Notes in Math. 340. Berlin-Heidelberg-New York: Springer 1973

11. Lipman, J.: Rational singularities ... Pub. Math. I.H.E.S. 36, 195-279 (1969)

12. Mazur, B., Messing, W.: Universal extensions and one dimensional crystalline cohomology. Lecture Notes in Math. 370. Berlin-Heidelberg-New York: Springer 1974

13. Messing, W.: The crystals associated to Barsotti-Tate groups. Lecture Notes in Math. 264. BerlinHeidelberg-New York: Springer 1972

14. Milne, J.S.: Duality in the flat cohomology of a surface. Ann. Sci. École Norm. Sup. (to appear)

15. Mumford, D.: Abelian varieties. Bombay: Oxford Press 1970

16. Raynaud, M.: Spécialisation du functeur de Picard. Publ. Math. I.H.E.S. 38, 27-76 (1970)

17. Serre, J.-P.: Groupes proalgébriques. Publ. Math. I.H.E.S. 7 (1960)

18. Seshadri, C.: L'opération de Cartier. Applications, Séminaire Chevalley, Varietés de Picard, Exposé 6, École Norm. Sup., Paris, 1958-59

Received February 25, 1976

M. Artin

Massachusetts Institute of Technology

Cambridge, Mass. 02139

USA

J.S. Milne

University of Michigan

Ann Arbor, Mich. 48109

USA 